\title{
Effet de l'inoculation ectomycorhizienne en pépinière sur la croissance et la nutrition des plants du cèdre de l'Atlas en Algérie
}

Hamida GabA-ChAHBoUB ${ }^{1}$

Mohammed S. LAMHAMEDI ${ }^{2}$

\section{Ouzna ABrous-BelbaCHIR ${ }^{3}$}

${ }^{1}$ Institut national de la recherche forestière Arboretum de Bainem (INRF Algérie) BP 37, Chéraga

Alger

Algérie

${ }^{2}$ Ministère des Forêts, de la Faune et des Parcs

Direction de la recherche forestière 2700 rue Einstein Québec G1P 3W8 Canada

${ }^{3}$ Université des sciences et de la technologie Houari Boumediene Faculté des sciences biologiques Laboratoire de Biologie et Physiologie des Organismes BP 32, El Alia

Bab Ezzouar, 16111

Alger

Algérie

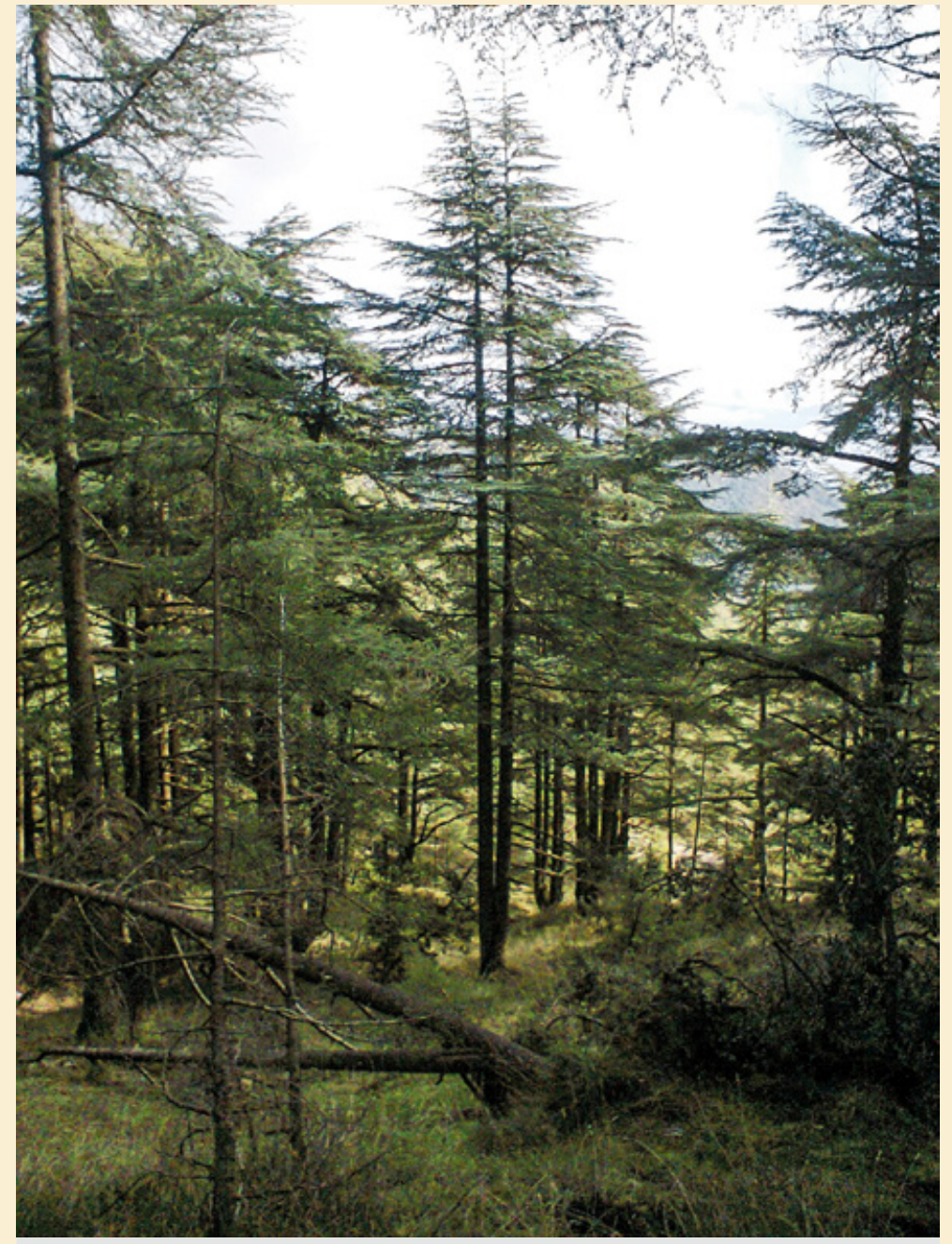

Photo 1.

Le cèdre de l'Atlas dans le Parc national de Chréa, en Algérie. Photo H. Chahboub, 2013. 


\section{RÉSUMÉ}

\section{EFFET DE L'INOCULATION ECTOMYCORHIZIENNE EN PÉPINIÈRE SUR LA CROISSANCE ET LA NUTRITION DES PLANTS DU CÈDRE DE L'ATLAS EN ALGÉRIE}

La mycorhization des plants du cèdre de l'Atlas en pépinière figure parmi les approches préconisées pour améliorer la survie et la croissance des plants en site de reboisement. Cependant, la majorité des travaux n'ont pas pu obtenir des taux de mycorhization élevés lorsque les plants du cèdre de l'Atlas étaient inoculés par des spores. L'objectif de cette étude est de déterminer l'influence de l'inoculum sporal de trois champignons ectomycorhiziens, Cortinarius cedretorum, Amanita vaginata et Inocybe geophylla, sur la croissance et la nutrition minérale des plantules de Cedrus atlantica. Les carpophores, les semences et le sol forestier organique utilisés pour la production des plants ont été prélevés sous un peuplement pur de cèdre de l'Atlas. L'inoculum sporal était constitué de carpophores broyés et tamisés. Après 12 mois de mise en culture, les différents paramètres de croissance ont été évalués : hauteur du plant, longueur de la racine principale, nombre de racines fines, taux de mycorhization, biomasse totale, nombre d'apex mycorhizés et concentrations foliaires des éléments minéraux. Les taux de mycorhization pour les trois champignons évalués ont varié entre $55 \%$ et $65 \%$, ce qui est un résultat exceptionnel dans le cas de l'inoculation des plants du cèdre de l'Atlas à l'aide des spores. Les résultats ont montré que l'inoculation avec les trois champignons ectomycorhiziens augmente significativement les concentrations foliaires de phosphore et d'azote. La biomasse des plantules du cèdre de l'Atlas est significativement supérieure avec les trois inocula comparativement aux plantules témoins.

Mots-clés : Cedrus atlantica, inoculum sporal, mycorhization, croissance, nutrition minérale, Algérie.

\section{ABSTRACT}

\section{EFFECTS OF ECTOMYCORRHIZAL INOCULATION ON GROWTH AND NUTRITION OF ATLAS CEDAR NURSERY SEEDLINGS IN ALGERIA}

Mycorrhizal inoculation of Atlas cedar seedlings in tree nurseries is one of the recommended technics for improving the survival and growth of trees in reforestation sites. However, most trials have not succeeded in obtaining high rates of mycorrhization in seedlings inoculated with spores. The aim of this study was to determine the influence of sporal inoculum from three species of ectomycorrhizal fungi, Cortinarius cedretorum, Amanita vaginata and Inocybe geophylla, on the growth and mineral nutrition of Cedrus atlantica seedlings. The carpophores, seeds and organic forest soils used to produce the seedlings were taken from beneath a pure stand of Atlas cedar. The sporal inoculum was made up of crushed and sieved carpophores. The different growth parameters were assessed after 12 months of culturing: seedling height, length of the main root, number of fine roots, mycorrhization rate, total biomass, number of mycorrhized root tips and concentrations of mineral elements in the leaves. Mycorrhization rates for the three fungi species assessed ranged from $55 \%$ to $65 \%$, which is exceptionally high for Atlas cedars inoculated with spores. The results show that inoculation with these three ectomycorrhizal fungi significantly increases leaf concentrations of phosphorus and nitrogen. Total biomass of the Atlas cedar seedlings was significantly higher with these three inocula than in the control seedlings.

Keywords: Cedrus atlantica, sporal inoculum, mycorrhization, growth, mineral nutrition, Algeria.

\section{RESUMEN}

\section{EFECTO DE LA INOCULACIÓN ECTOMICORRÍCICA EN VIVERO SOBRE EL CRECIMIENTO Y NUTRICIÓN DE PLÁNTULAS DE CEDRO DEL ATLAS EN ARGELIA}

La micorrización de plántulas de cedro del Atlas en vivero es uno de los métodos recomendados para mejorar la supervivencia y crecimiento de las plantas en zonas de repoblación. Sin embargo, la mayoría de estudios no lograron obtener altas tasas de micorrización cuando se inoculaban las plantas de cedro del Atlas con esporas. Este estudio intenta determinar la influencia del inóculo esporal de tres hongos ectomicorrícicos, Cortinarius cedretorum, Amanita vaginata e Inocybe geophylla, en el crecimiento y nutrición mineral de las plántulas de Cedrus atlantica. Los carpóforos, semillas y suelo forestal orgánico empleados en la producción de las plántulas se tomaron en un rodal puro de cedro del Atlas. El inóculo esporal estaba compuesto por carpóforos molidos y tamizados. Tras 12 meses de cultivo, se evaluaron los diferentes parámetros de crecimiento: altura de la planta, longitud de la raíz principal, número de raíces finas, tasa de micorrización, biomasa total, número de ápices micorrizados y concentraciones foliares de elementos minerales. Los porcentajes de micorrización de los tres hongos evaluados variaron entre el $55 \%$ y el $65 \%$; siendo este un resultado excepcional en el caso de la inoculación de plantas de cedro del Atlas mediante esporas. Los resultados mostraron que la inoculación con los tres hongos ectomicorrícicos aumenta significativamente las concentraciones foliares de fósforo y nitrógeno. La biomasa de las plántulas de cedro del Atlas es significativamente mayor con los tres inóculos que en las plántulas testigo.

Palabras clave: Cedrus atlantica, inóculo esporal, micorrización, crecimiento, nutrición mineral, Argelia. 
Introduction

Le cèdre de l'Atlas (Cedrus atlantica Manetti Endl.) figure parmi les essences les plus productives de la région méditerranéenne, notamment en Algérie et au Maroc (M'Hirit, 1994). En Algérie, le cèdre couvre 29000 hectares (Derridj, 1990), soit $1,24 \%$ de la superficie forestière du pays. Cette essence noble est fortement recherchée pour ses nombreuses utilisations (sciage, charbon, goudron, résine, tanin, etc.).

La régénération naturelle des arbres dans les différents peuplements du cèdre est affectée négativement par des facteurs de dégradation (surpâturage, coupes illicites, incendies, insectes et maladies, etc.) et par l'augmentation de la fréquence des périodes de sécheresse (Zine El Abidine et al., 2013). La sévérité de ces sécheresses augmente l'intensité du stress hydrique et par conséquent le dépérissement des arbres matures et la mortalité des jeunes plants de cette essence au Maroc et en Algérie (Bentouati et Bariteau, 2006 ; Zine El Abidine et al., 2013). Ainsi, des efforts ont été consentis en matière de reboisement pour la sauvegarde, la restauration, la conservation et l'extension du cèdre de l'Atlas en Algérie dans son aire écologique. Cependant, après la mise en terre, les taux de mortalité des plants sont relativement élevés pendant les premières années de croissance dans les différents périmètres de reboisement. En plus de la sévérité des stress environnementaux, cette mortalité dans les périmètres de reboisement est fortement liée à la mauvaise qualité morpho-physiologique des plants produits dans les pépinières forestières (Lamhamedi et al., 2000). Avec les changements climatiques anticipés dans la zone méditerranéenne, l'Afrique du Nord sera davantage touchée par un réchauffement compris entre $3^{\circ} \mathrm{C}$ et $4^{\circ} \mathrm{C}$ (FAO, 2013). Ces changements pourraient se traduire par des étés plus chauds et secs et des événements extrêmes plus marqués (durée longue des sécheresses, augmentation de la fréquence des feux, attaques d'insectes et maladies, etc.), ainsi que par une diminution des précipitations qui pourrait aller jusqu'à $20 \%$ (FAO, 2013).

Face à cette situation, l'amélioration du taux de survie des plants dans les périmètres de reboisement en Afrique du Nord ne peut se concrétiser que par l'utilisation de plants de haute qualité morpho-physiologique pourvus d'un excellent système racinaire. Le recours à la mycorhization figure parmi les techniques à privilégier pour améliorer davantage la qualité du système racinaire et la survie des plants tout en leur conférant une tolérance accrue à la sécheresse et aux différentes contraintes des sites de reboisement méditerranéens (Lamhamedi et al., 1991 ; Mousain et al., 1994). À cet effet, en Afrique du Nord, des efforts ont été consentis en matière de modernisation des pépinières forestières. Cependant, l'accent a été davantage mis sur l'installation des infrastructures modernes (conteneurs, ombrières, automatisation de l'irrigation et de la fertilisation, etc.), le compostage, et la mycorhization contrôlée à l'aide de Rhizopogon sp. et de Pisolithus tinctorius (Lamhamedi et al., 2000, 2009). Ces champignons ectomycorhiziens ont été utilisés également pour corriger la carence en fer chez les plants de certaines essences résineuses (pin d'Alep, pin pignon, cyprès, etc.) (Lamhamedi et al., 2009).
Par contre, les plants du cèdre de l'Atlas n'ont pas fait l'objet de production dans les pépinières modernes combinée à la mycorhization à une échelle opérationnelle car il est très difficile d'obtenir un taux de mycorhization supérieur à 50 \% (Abourouh, 2000 ; Lamhamedi et al., 2009). À titre d'exemple, dans les pépinières forestières, la proportion de plants du cèdre qui possèdent naturellement des ectomycorhizes dépasse rarement 4 \% (Abourouh, 2000). Par ailleurs, Marx (1979) a observé que le taux de mycorhization des racines du cèdre dans plusieurs pépinières forestières varie entre 10 et $20 \%$, bien que le cèdre forme des associations symbiotiques avec plus de 15 champignons ectomycorhiziens (Nezzar Hocine, 1998 ; Abourouh, 2000). Boukcim et al. (2002) ont utilisé deux types d'inoculum (solide sur tourbe-vermiculite ou par inclusion dans l'alginate) de Tricholoma tridentinum var. cedretorum et Hebeloma sinapizans, mais le taux de mycorhization a rarement dépassé $50 \%$. La difficulté de mycorhization du cèdre au stade juvénile est probablement liée à la non-réceptivité des racines et à l'utilisation des isolats sous forme sporale ou végétative, de champignons non spécifiques et non collectés sous des peuplements naturels de cette essence (Mousain et al., 1987). L'absence d'une mycorhization abondante chez les plants du cèdre de l'Atlas pourrait également être due à une incompatibilité entre les deux génotypes (champignon ectomycorhzien-arbre), non favorable à la colonisation et au développement des ectomycorhizes et de la phase extramatricielle (Lamhamedi et al., 1990, 1991).

Dans le cas du cèdre de l'Atlas, les données sur la mycorhization avec des spores récoltées dans le même site et leur éventuel comportement en pépinière forestière restent fragmentaires et peu nombreuses (Abourouh, 2000 ; Lamhamedi et al., 2009). À cet effet, nous supposons que le recours à l'utilisation de spores de champignons ectomycorhiziens, de graines du cèdre de l'Atlas et du sol organique récoltés dans les mêmes peuplements forestiers peut contribuer à favoriser la mycorhization abondante au stade juvénile des plants du cèdre. Dans ce sens, l'inoculation sporale est une technique peu coûteuse, à la portée du pépiniériste et facile à intégrer à l'échelle opérationnelle en pépinière forestière. De plus, à l'inverse des inocula solides et liquides où généralement une seule souche (= un génotype) est utilisée, l'inoculation sporale permet l'introduction d'une diversité de génotypes du champignon et l'augmentation de la probabilité de combinaisons compatibles des génotypes (champignon-cèdre) qui peut favoriser l'obtention d'une bonne colonisation des racines. Dans le cadre de cette étude, trois champignons ectomycorhiziens (Cortinarius cedretorum R. Maire, Amanita vaginata (Fries) Vittidini et Inocybe geophylla (Pers.) P. Kumm) ont été utilisés à cause de la formation d'ectomycorhizes avec les racines des arbres et de l'abondance de leurs carpophores sous les peuplements purs du cèdre de l'Atlas. À notre connaissance, il s'agit de la première étude qui combine l'utilisation des spores issues de plusieurs carpophores, des graines et du sol échantillonnés sous le même peuplement. Ainsi, les objectifs de ce travail consistent à évaluer, d'une part, la colonisation des racines des plants du cèdre 
de l'Atlas en réponse à des inocula sporaux de trois champignons ectomycorhiziens (Cortinarius cedretorum, Amanita vaginata et Inocybe geophylla) et, d'autre part, les effets de ces champignons ectomycorhiziens sur la croissance et les concentrations en éléments nutritifs (phosphore et azote) des plants du cèdre de l'Atlas.

\section{Matériel et méthodes}

\section{Site d'étude}

Les carpophores des champignons ectomycorhiziens, le sol et les graines du cèdre de l'Atlas ont été collectés dans le même peuplement du Parc national de Chréa ( $36^{\circ} 25^{\prime} \mathrm{N}$, $2^{\circ} 52^{\prime} \mathrm{E}$; altitude : $1600 \mathrm{~m}$; précipitations : $1400 \mathrm{~mm} /$ an) localisé dans l'Atlas tellien occidental de l'Algérie où les vents dominants sont de type nord-ouest et humides. La période pluvieuse est de dix mois (septembre à juin) et la période sèche dure deux mois (juillet et août). Les températures maximales moyennes du mois le plus chaud varient entre $26,3{ }^{\circ} \mathrm{C}$ et $33,6^{\circ} \mathrm{C}$, et les températures minimales moyennes du mois le plus froid oscillent entre $0,4^{\circ} \mathrm{C}$ et $7,3^{\circ} \mathrm{C}$. Les analyses texturale et physicochimique du sol forestier utilisé dans le cadre de cette étude ont révélé une texture limono-argilo-sableuse (argile : 13,95\% ; limon fin : $20,3 \%$; limon grossier : $25,22 \%$; sable fin : $2,32 \%$; sable grossier : 38,21\%), légèrement acide, riche en matière organique et peu calcaire (carbone total : 5,25\% ; azote total : $0,08 \%$; matière organique : $9,12 \% ; \mathrm{pH}_{\text {eau }}: 5,9$; calcaire total : 1,2\% ; conductivité électrique : $2,9 \mathrm{DS} / \mathrm{cm})$. Le phosphore assimilable a été dosé selon la méthode décrite par Olsen et al. (1954). La concentration en phosphore sous forme $\mathrm{P}_{2} \mathrm{O}_{5}$ était de $0,9 \mathrm{mg} / \mathrm{kg}$ de sol.

\section{Germination des graines}

Les graines de la provenance du cèdre du Parc national de Chréa ont été récoltées à partir de 10 arbres semenciers distants de $20 \mathrm{~m}$ l'un de l'autre. Les graines ont été stérilisées en surface avec du peroxyde d'hydrogène $\left(\mathrm{H}_{2} \mathrm{O}_{2}\right)$ à $30 \%$ pendant $5 \mathrm{~min}$, rincées trois fois à l'eau distillée et ensuite stratifiées à $4{ }^{\circ} \mathrm{C}$ pendant deux semaines à l'obscurité en conditions semi-axéniques. Elles ont été transférées dans des boîtes de Petri tapissées de papier filtre humide et mises à germer dans une chambre de croissance à $25^{\circ} \mathrm{C}$ jour/nuit à $65 \%$ de taux d'humidité.

\section{Préparation de l'inoculum ectomycorhizien}

Trois champignons ectomycorhiziens ont été récoltés pendant le mois de janvier 2013 dans le même site de collecte des semences. Il s'agit de Cortinarius cedretorum (CC, 20 carpophores), Amanita vaginata (AV, 32 carpophores) et Inocybe geophylla (IG, 28 carpophores). Chaque champignon se distingue par des caractéristiques morphologiques qui lui sont propres.

- Amanita vaginata : chapeau gris, gris ocracé, conique et enfin plan et mamelonné, brillant, à marge longuement striée ; lames peu serrées, blanches, arêtes " givrées »; stipe non bulbeux, creux et fragile, blanchâtre, chiné ou non de gris et engainé dans une volve profonde, blanche et une chair fragile, blanche.

- Inocybe geophylla : chapeau de 2 à $5 \mathrm{~cm}$, conique pointu s'étalant avec mamelon marqué, lisse et soyeux, à marge légèrement striée se fendillant souvent, de couleur lilas à violet clair ; lames adnées, doublées de lamelles ou lamellules, de couleur crème à beige puis brunissant ; pied fibreux, parfois courbé, blanchâtre à beige grisâtre, parfois légèrement violacé ; se développant le plus souvent en groupes de plusieurs individus.

- Cortinarius cedretorum : chapeau ocre jaune olivâtre à centre roussâtre ; lames olivâtres claires ; pied à bulbe marginé ; chair violacée dans toutes ses parties, plus jaune dans le bulbe.

Après leur identification avec un numéro de laboratoire à des fins de conservation, les carpophores frais ont été nettoyés délicatement, puis séchés à l'air libre, puis à l'étuve à $30^{\circ} \mathrm{C}$ pendant $24 \mathrm{~h}$ pour enlever toute trace d'humidité. Le nettoyage était effectué à l'aide d'une petite brosse afin d'éliminer les fragments de racines et les particules du sol susceptibles d'introduire des contaminants microbiens. Par la suite, ils ont été moulus à l'aide d'un broyeur pendant une très courte période ( $2 \mathrm{~min})$. L'inoculum sporal a été tamisé à l'aide d'un tamis dont les mailles avaient un diamètre de $200 \mu \mathrm{m}$. Cet inoculum sporal sous forme d'une suspension a été maintenu au réfrigérateur $\left(4^{\circ} \mathrm{C}\right)$ jusqu'à son utilisation.
Photo 1.

Le cèdre de l'Atlas dans le Parc National de Chréa, en Algérie. Photo H. Chahboub, 2013. 


\section{Substrat de culture}

Le substrat de culture utilisé était un échantillon composite du sol rhizosphérique (30 kg) prélevé au niveau de l'horizon A1 du cèdre, au pied de 10 arbres adultes et sains du même site de collecte des graines et des carpophores, au sein du Parc national de Chréa.

Avant son utilisation et afin d'éliminer la microflore indigène, le sol a été tamisé à l'aide d'un tamis de $2 \mathrm{~mm}$ et stérilisé deux fois à $120^{\circ} \mathrm{C}$ pendant $48 \mathrm{~h}$ d'intervalle.

\section{Méthode d'inoculation et conditions de culture}

L'expérimentation a été menée en serre expérimentale de l'Institut national de la recherche forestière (arboretum de Bainem à l'ouest d'Alger, $\left.36^{\circ} 45^{\prime} \mathrm{N}, 3^{\circ} 25^{\prime} \mathrm{E}\right)$. Le dispositif expérimental à randomisation aléatoire comprenait quatre traitements : trois traitements sur des plantules du cèdre de l'Atlas inoculées chacune par Cortinarius cedretorum (CC), Amanita vaginata (AV) et Inocybe geophylla (IG), et un traitement témoin sur des plantules témoins non inoculées. Pour chaque traitement, le nombre de répétitions était de 20 plantules distribuées aléatoirement.

Après un mois de mise en culture dans des conteneurs Riedacker (hauteur : $17 \mathrm{~cm}$; diamètre de la section : $10 \mathrm{~cm}$ ) à section WM (Thermoflan, Molières-Cavaillac, Le Vigan, France), chaque plant a été inoculé à l'aide d'un inoculum sporal constitué d'un mélange de $0,1 \mathrm{~g}$ de broyat tamisé des carpophores et de $250 \mathrm{ml}$ d'eau distillée. Chaque cavité du conteneur contenait à la fois les racines du plant et le substrat ; l'inoculum a été placé à $4 \mathrm{~cm}$ de profondeur en contact direct avec les racines (Abourouh, 1995). Le substrat a été ramassé sous un peuplement forestier du cèdre de l'Atlas au Parc national de Chréa, dont les propriétés physico-chimiques sont indiquées dans la section consacrée au site d'étude. Les plantules ont été maintenues à une photopériode de $16 / 8 \mathrm{~h}$, et à une température de $25 \pm 2{ }^{\circ} \mathrm{C}$ le jour et $18 \pm 2{ }^{\circ} \mathrm{C}$ la nuit à $65 \%$ d'humidité relative.

Les plants ont été arrosés trois fois par semaine avec de l'eau distillée stérile pour maintenir des teneurs en eau du sol proches de la saturation afin de favoriser la croissance des plants et du champignon. Le contrôle manuel des mauvaises herbes a été effectué au fur et à mesure tout au long de la durée de l'expérience.

\section{Analyse minérale des aiguilles}

Après séchage à l'étuve à $65^{\circ} \mathrm{C}$, les concentrations en azote et en phosphore des aiguilles de chaque plantule (1 g d'aiguilles sèches/échantillon) du cèdre de l'Atlas, âgées de 12 mois, ont été déterminées par la méthode de Kjeldahl (John, 1970) pour l'azote tandis que le phosphore a été dosé par la méthode colorimétrique au bleu molybdène (Murphy et Riley, 1962).

\section{Évaluation des paramètres de croissance}

Pour chaque plant, nous avons mesuré la hauteur, la longueur de la racine principale, le nombre d'apex mycorhizés et la masse sèche totale après un séchage à l'étuve à $65{ }^{\circ} \mathrm{C}$ pendant $48 \mathrm{~h}$; 20 plantules/traitement ont été utilisées, soit un total de 80 plantules.

\section{Étude morphologique et anatomique des ectomycorhizes}

Tous les plants ont été examinés quant à la présence ou l'absence de la colonisation ectomycorhizienne des racines fines. Le taux de mycorhization a été déterminé sur l'ensemble du système racinaire comme étant le nombre d'ectomycorhizes / nombre total de racines fines x 100 (Dupponnois et al., 2005).

Après lavage des racines de tous les plants sous un filet d'eau du robinet, la typologie des racines mycorhizées a été déterminée sous une loupe stéréoscopique ( $\mathrm{G} 8 \mathrm{x}$ ) selon différents caractères morphologiques : forme générale, mode de ramification, structure et épaisseur du manteau fongique (Agerer, 1995). Ensuite, des échantillons représentatifs de chaque type d'ectomycorhize ont été photographiés, puis fixés dans un mélange de formol, acide acétique et alcool (90-5-5) Johansen, 1940), et conservés à température ambiante. L'étude anatomique a été réalisée à l'aide de coupes transversales effectuées à main levée, puis colorées avec du bleu de coton (0,05\%) et du lactophénol de Amann.

\section{Analyses statistiques}

Les données morphologiques obtenues ont fait l'objet d'une analyse de la variance (Anova) et les moyennes ont été comparées à l'aide du test multivarié de Newman et Keuls $(P<0,05)$. Les coefficients de corrélation de Pearson ont été calculés pour l'estimation de la relation entre le taux de mycorhization et les paramètres de croissance évalués pour un risque d'erreur de $5 \%$.

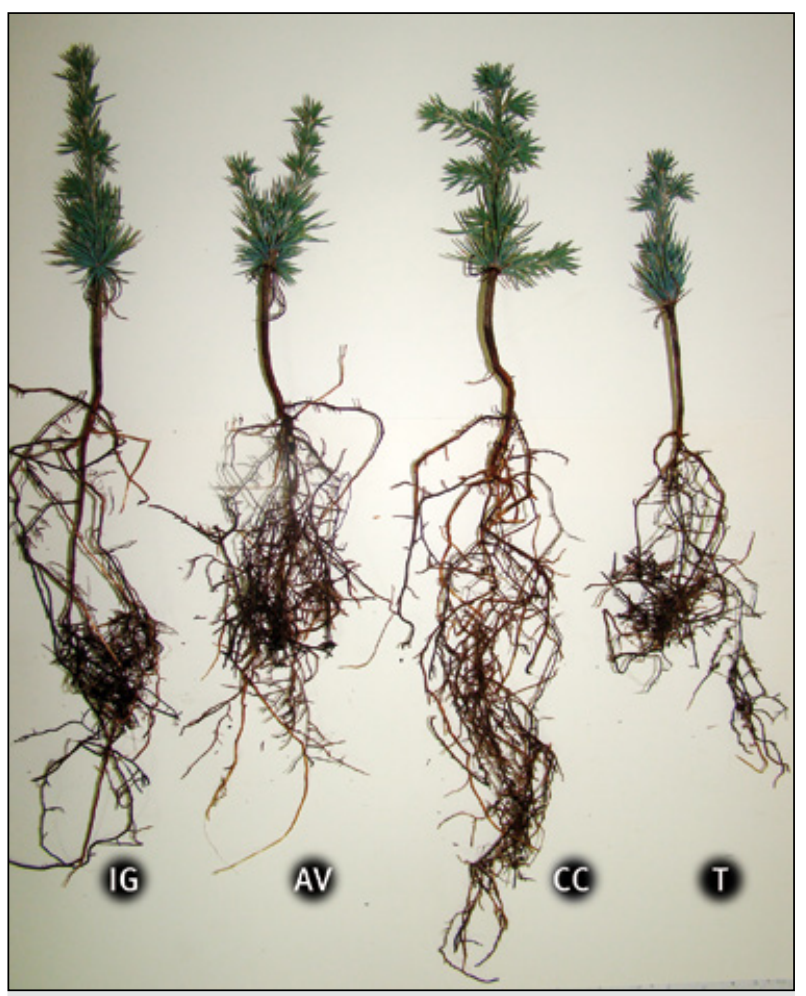

Figure 1.

Effet de l'inoculation avec les spores de trois champignons ectomycorhiziens sur la morphologie des plantules du cèdre de l'Atlas âgées de 12 mois. IG : Inocybe geophylla; AV : Amanita vaginata ; CC : Cortinarius cedretorum; $\mathrm{T}$ : témoin. 


\section{Résultats}

\section{Effet de la mycorhization sur la croissance des plantules du cèdre de l'Atlas}

En comparaison avec les plantules témoins, les plantules inoculées présentaient des parties aériennes plus développées et des chevelus racinaires plus ramifiés (figure 1).

Tous les plants du cèdre de l'Atlas inoculés ont formé des apex mycorhizés alors que ce n'est pas le cas pour les plants témoins. En effet, l'inoculation avec les spores des deux champignons ectomycorhiziens CC et $\mathrm{AV}$ a donné des nombres d'apex mycorhizés relativement élevés $(123,1 \pm 14,04 ; 115,5 \pm 10,62)$ avec des taux de mycorhization similaires $(65,01 \%$ et $64,2 \%$ respectivement) (figure $2 \mathrm{a}$ ). Ces taux restent significativement différents $(p<0,05)$ de ceux d'IG, dont le nombre d'apex mycorhizés est de 81,7 $\pm 13,4$ et le taux de mycorhization de 54,9\% (figure $2 a$ et $b$ ). Par ailleurs, le nombre de racines fines des plants témoins est de $3,6 \pm 1,5$; ce nombre augmente pour atteindre $6,5 \pm 1,9,9,5 \pm 1,64$ et $21 \pm 2,74$ respectivement pour les inocula IG, AV et CC (figure $2 \mathrm{c}$ ).

La longueur de la racine principale était significativement plus élevée en présence des trois inocula, comparativement aux plants témoins. En effet, les champignons CC, AV et IG ont significativement augmenté la longueur de

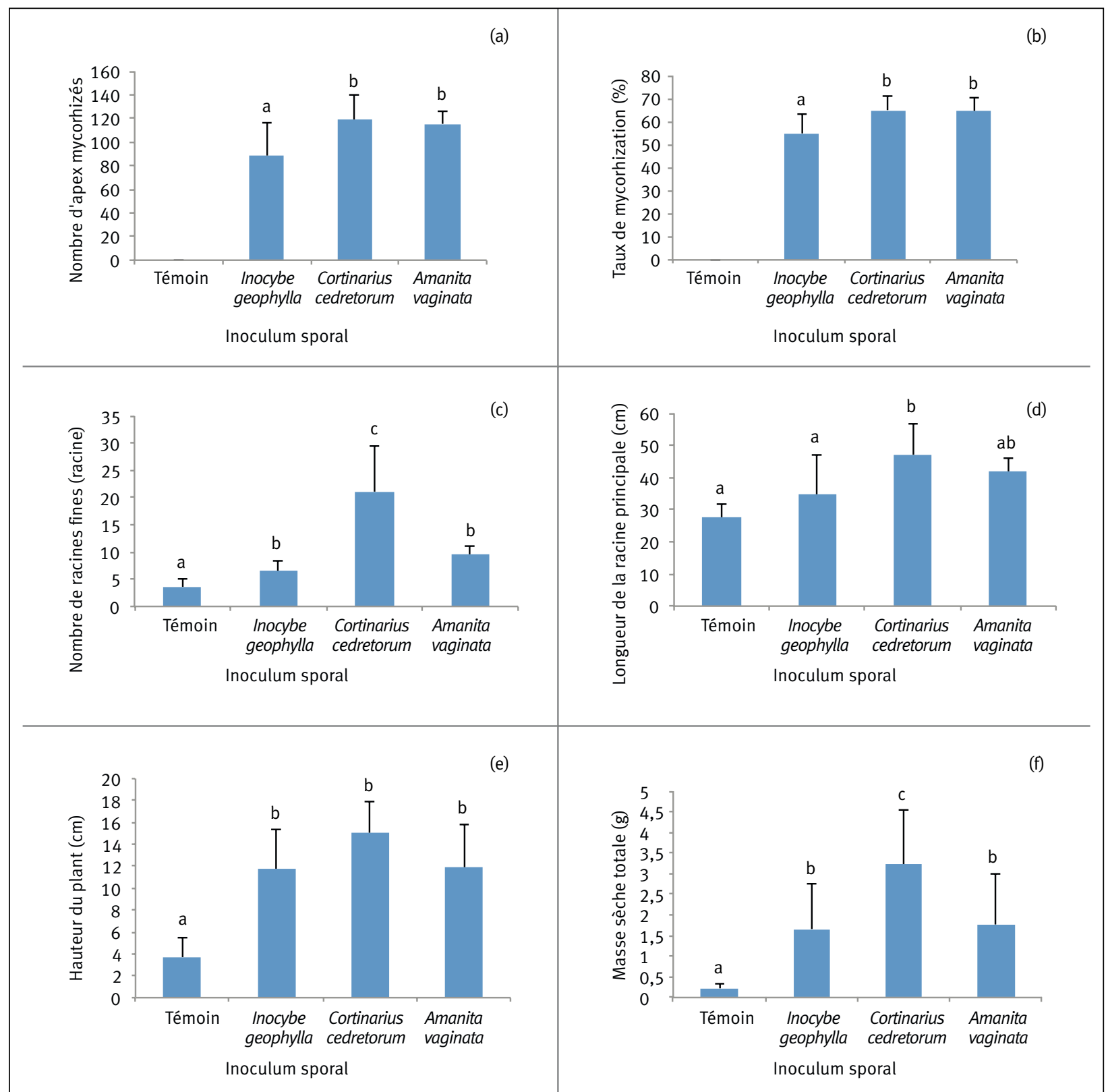

Figure 2.

Paramètres de croissance et de mycorhization des plantules du cèdre de l'Atlas témoins et inoculées par trois champignons ectomycorhiziens. a : nombre d'apex mycorhizés ; b : taux de mycorhization ; c : nombre de racines fines ; $d$ : longueur de la racine principale ; $\mathrm{e}$ : hauteur du plant; $\mathrm{f}$ : masse sèche totale du plant. Pour chaque champignon ectomycorhizien, les barres verticales indiquent les erreurs standards $(n=20)$. Pour chaque paramètre, les lettres différentes indiquent des différences significatives entre les trois traitements selon le test de Newman-Keuls $(P<0,05)$. 
la racine principale respectivement de $85,81 \%$, $52 \%$ et $24,83 \%$ (figure 2 d). Les valeurs moyennes observées pour la croissance en hauteur des plants ont atteint 15,95 $\pm 2,08$, $14,1 \pm 2,28$ et $11,11 \pm 2,14 \mathrm{~cm}$ chez les plants inoculés respectivement par CC, AV et IG, par comparaison aux plants témoins (figure 2e).

Par ailleurs, les plants inoculés avec CC ont manifesté les plus hautes valeurs des masses sèches totales par rapport aux autres inocula AV et IG ; cette augmentation était de $82 \%, 75 \%$ et $57 \%$ pour les trois inocula respectifs (CC, AV, IG) (figure 2f).

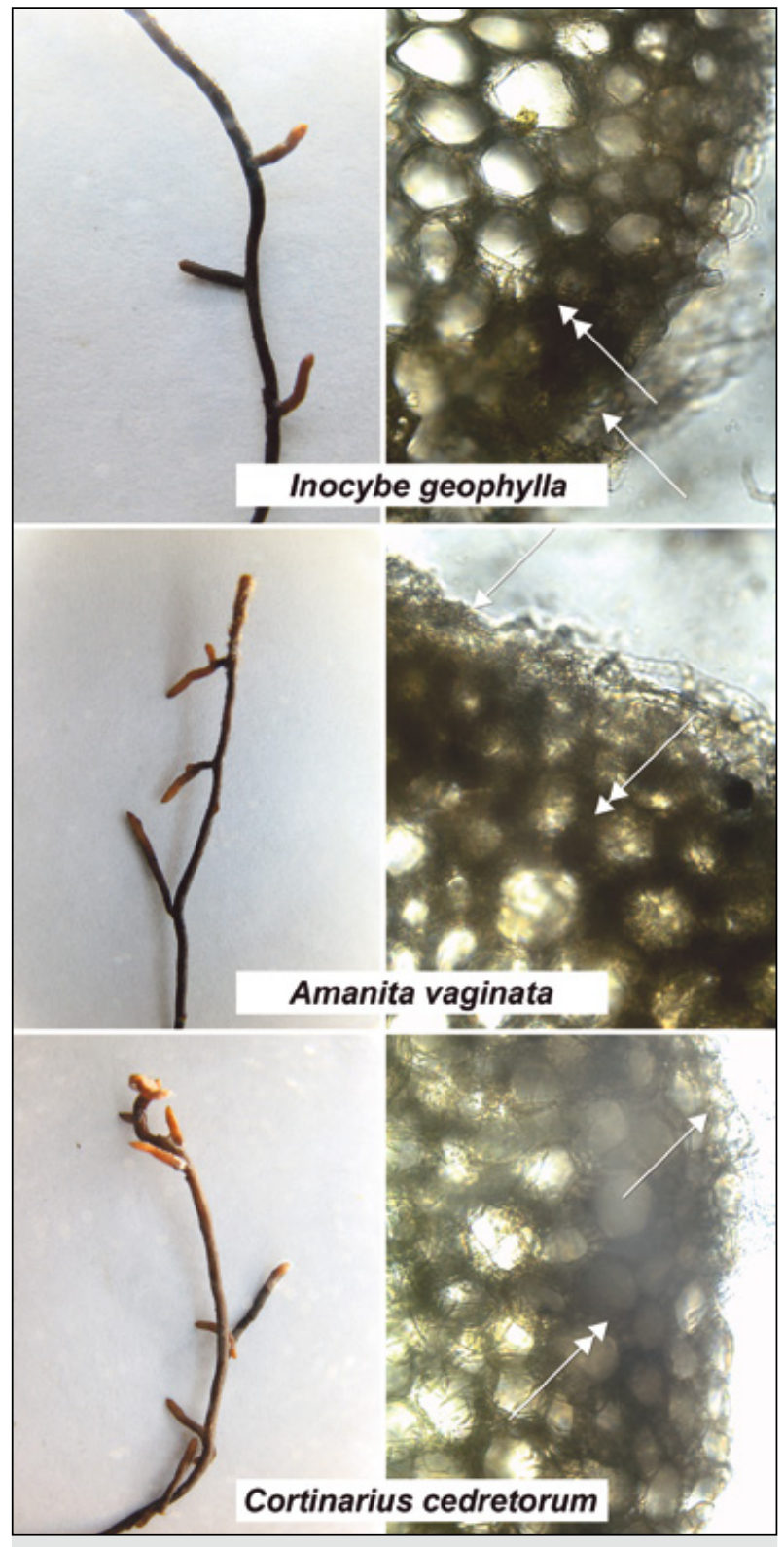

Figure 3.

Observation à la loupe stéréoscopique $(\mathrm{G} \times \mathrm{8})$ des morphotypes des apex mycorhizés des plantules inoculées âgées de 12 mois et des coupes anatomiques au microscope photonique ( $\mathrm{G} \times 400)$. La flèche indique le manteau fongique tandis que la double flèche montre le réseau de Hartig.

\section{Morphotypes et coupes anatomiques des apex mycorhizés}

L'analyse macroscopique à la loupe stéréoscopique a révélé trois morphotypes d'apex ectomycorhizés. Ils sont dichotomiques à surface lisse de couleur marron-noir de 3 à $5 \mathrm{~mm}$ de longueur chez IG. Ils sont également dichotomiques à surface lisse de couleur brun foncé, parfois noire, et de $3 \mathrm{~mm}$ de longueur chez AV. Les morphotypes formés par CC sont monopodiaux pennés à surface lisse, de $3 \mathrm{~mm}$ de longueur, à ramification simple et de couleur marron-noir (figure 3).

D'autre part, les observations microscopiques ont révélé la présence d'un manteau fongique bien visible et un réseau de Hartig bien développé avec absence du rhizomorphe pour les trois inocula utilisés; la taille faible du manteau fongique a caractérisé les trois types d'apex des inocula CC, AV, IG ; le réseau de Hartig était moyennement profond, avec trois à quatre assises cellulaires du parenchyme cortical (figure 3).

\section{Concentrations en phosphore et en azote des aiguilles des plantules du cèdre}

Le dosage du phosphore et de l'azote montre que chez les plantules témoins la concentration moyenne en phosphore était de 145,33 $\pm 3,05 \mu \mathrm{g} / \mathrm{g}$ MVS (MVS : matière végétale sèche) ; cette concentration s'élevait à $418 \pm 2,38(\mu \mathrm{g} / \mathrm{g}$ MVS), 438,4 \pm 4,82 ( $\mu \mathrm{g} / \mathrm{g}$ MVS) et $482 \pm 2,73$ ( $\mu \mathrm{g} / \mathrm{g}$ MVS) (figure 4a) pour les trois inocula respectifs IG, AV et CC, ce qui montre que le gain en phosphore des aiguilles des plants inoculés était trois fois plus grand que celui des témoins. Pour l'azote, une concentration moyenne de 16,33 $\pm 1,52(\mu \mathrm{g} / \mathrm{g}$ MVS) a été relevée chez les plantules témoins, cette concentration s'accroissant pour atteindre 38,2 $\pm 3,70$ ( $\mu \mathrm{g} / \mathrm{g} \mathrm{MVS}$ ), $48,8 \pm 3,56$ ( $\mu \mathrm{g} / \mathrm{g}$ MVS) et $49 \pm 2,33$ ( $\mu \mathrm{g} / \mathrm{g}$ MVS) (figure $4 \mathrm{~b}$ ), soit des augmentations de deux fois pour l'inoculum IG et de trois fois pour les inocula CC et AV par rapport aux plantules témoins. Cependant, nous n'avons observé aucune différence significative entre les inocula CC et AV, alors que la différence était significative entre IG et les deux inocula $C C$ et $A V$.

\section{Corrélation des paramètres de croissance avec le taux de mycorhization}

Les résultats de la matrice de corrélation entre les différents paramètres morphologiques et le taux de mycorhization ont révélé un haut niveau de corrélation. Ainsi, les coefficients de détermination de ces corrélations étaient de $0,66,0,89$ et 0,94 respectivement pour les paramètres suivants : longueur de la racine principale, nombre d'apex mycorhizés et nombre de racines fines (figure 5). 


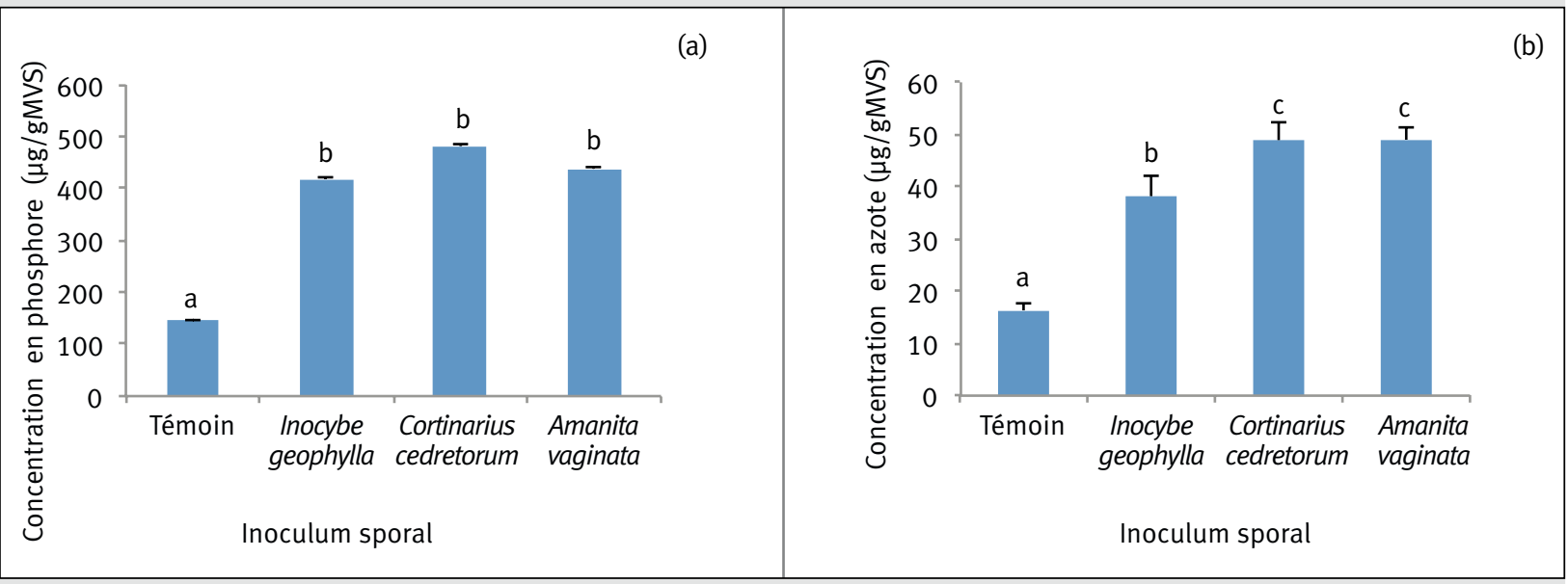

Figure 4.

Effet de l'inoculation avec les spores de trois champignons ectomycorhiziens sur les concentrations en phosphore (a) et en azote (b) des aiguilles de plantules du cèdre de l'Atlas âgées de 12 mois. Pour chaque champignon ectomycorhizien, les barres verticales indiquent les erreurs standard $(n=20)$. Pour chaque paramètre, les lettres différentes indiquent des différences significatives entre les trois traitements selon le test de Newman-Keuls $(P<0,05)$.

\section{Discussion}

Les résultats de cette étude ont révélé une variabilité des taux de mycorhization (54,9-65,01 \%) des plantules inoculées avec les inocula sporaux utilisés (IG, AV et CC), ainsi qu'une amélioration de la croissance et de la nutrition azotée et phosphatée des plantules du cèdre de l'Atlas. À notre connaissance, c'est la première fois que la mycorhization des plants du cèdre de l'Atlas à l'aide de spores fut un succès, et que le taux de mycorhization a dépassé $50 \%$. Jusqu'à présent, seul le recours à l'inoculum solide avait permis d'obtenir des taux de mycorhization des plants du cèdre de l'Atlas de 50 \% (Boukcim et al., 2002 ; Nezzar Hocine, 1998). Plusieurs travaux ont montré que le taux de mycorhization des plants du cèdre de l'Atlas à l'aide de spores variait entre 4 et $20 \%$ (Abourouh, 1993, 2000 ; Marx, 1979).

L'obtention de ces taux de mycorhization relativement élevés laisse présager que ce résultat est l'expression de l'interaction de l'utilisation de spores de champignons (IG,
$\mathrm{AV}$ et $\mathrm{CC}$ ) en association naturelle avec les arbres du cèdre, des graines récoltées sur des arbres semenciers, et du terreau forestier ramassé sous des peuplements du cèdre de l'Atlas de la même région écologique. À l'inverse de l'utilisation d'une seule souche de génotype connu (inoculum solide, liquide, etc.), le recours à l'inoculum sporal permet aux racines du cèdre de l'Atlas d'être mycorhizées avec une diversité de génotypes (plusieurs monocaryons et dicaryons) (Lamhamedi et al., 1990) du champignon (IG, AV et $\mathrm{CC})$. Cette approche augmente la probabilité de compatibilité entre les génotypes de la plante hôte et du champignon ectomycorhizien (Lamhamedi et al., 1990, 1991 ; Rosado et al., 1994a, 1994b) à l'aide d'échanges de signaux qui induisent des changements d'expression du génome des deux symbiotes (Wong et Fortin, 1990). Cette diversité de souches de la même espèce en association symbiotique avec les racines du cèdre de l'Atlas contribue à conférer à la plante hôte une tolérance accrue aux stress environnementaux en site de plantation (Lamhamedi et al., 1991). La

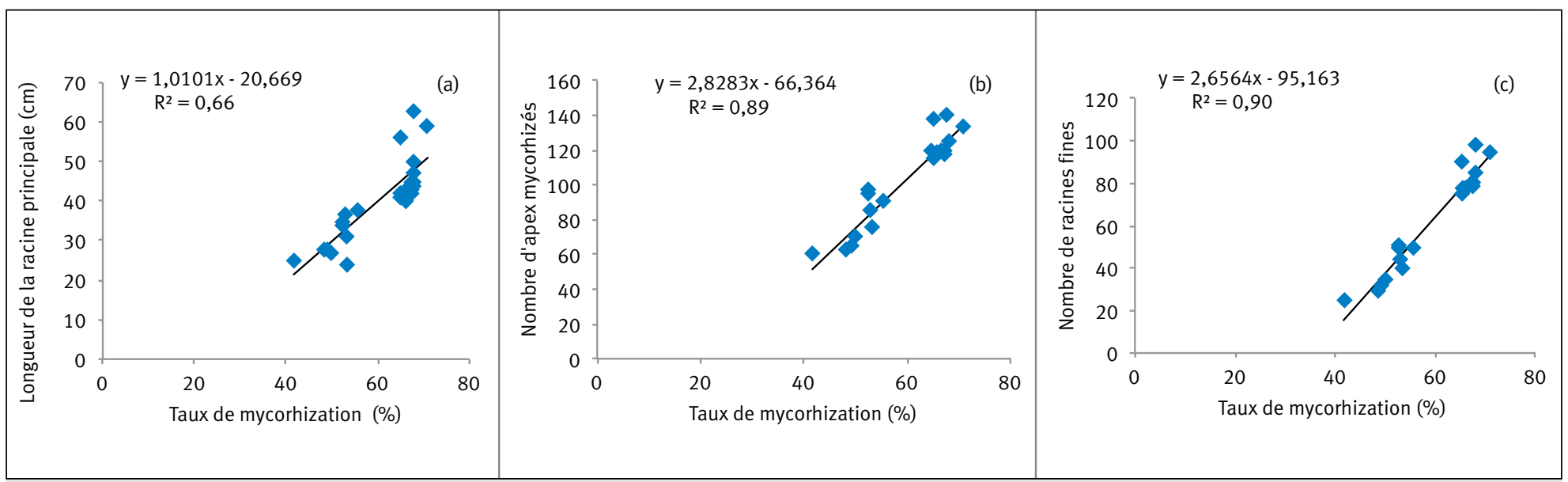

Figure 5.

Relations linéaires entre la longueur de la racine principale (a), le nombre d'apex mycorhizés (b), le nombre de racines fines (c) et le taux de mycorhization. 
mycorhization est également influencée par les propriétés physico-chimiques du sol ou du terreau forestier, notamment la texture, la fertilité, le pH, l'aération (Sanon et al., 2011) et les exsudats racinaires (Theodorou et Bowen, 1987). L'utilisation des inocula de champignons de stade précoce pourrait augmenter davantage les taux de mycorhization des plants du cèdre de l'Atlas, contrairement aux inocula de champignons tardifs dont font partie les champignons AV, IG et CC (Guinberteau et Courtecuisse, 1997).

Les différences observées entre les degrés de mycorhization seraient aussi attribuables à des différences en matière d'interactions (cèdre-champignon) qui affectent le pouvoir mycorhizogène de chaque espèce ectomycorhizienne vis-à-vis des racines des plantules de cèdre. Cette hypothèse a été démontrée dans diverses associations ectomycorhiziennes (Torres et Honrubia, 1994 ; Conjeaud et al., 1996).

Les trois inocula sporaux utilisés, CC, AV et IG, ont favorisé la croissance du cèdre. Les plants inoculés ont montré une hauteur, une longueur de la racine principale et une masse sèche totale significativement supérieures respectivement à celles des plantules témoins.

De nombreux travaux ont mis en évidence l'effet bénéfique de l'inoculation contrôlée par divers champignons ectomycorhiziens sur la croissance des plantes hôtes, Pinus halepensis avec une suspension sporale de Suillus collinitus (Rincón et al., 2007) et Eucalyptus avec une suspension sporale de Scleroderma (Chen et al., 2006). Cependant, on ne dispose que de très peu de travaux traitant de la mycorhization du cèdre de l'Atlas avec des suspensions sporales, des graines et du terreau issus du même peuplement du cèdre.

Les résultats obtenus sont en accord avec ceux de Mousain et al. (1987) qui ont révélé une stimulation de la croissance en hauteur de l'ordre de $45 \%$ de jeunes cèdres inoculés en pépinière par une suspension sporale de Tuber albidum. Ce résultat a été confirmé après quatre années de croissance dans des plantations du cèdre dans son environnement naturel.

En Algérie, Nezzar Hocine (1998) a réalisé un inventaire mycologique sur les champignons ectomycorhiziens associés au cèdre de l'Atlas du Parc national de Djurdjura ; elle a démontré que la flore mycorhizienne du cèdre de l'Atlas dans une plantation de cèdre des montagnes du Djurdjura est constituée particulièrement par les espèces appartenant aux genres Cortinarius, Inocybe, Russula et Tricholoma. Dans ce travail, les mycorhizes observées avec les racines des arbres et les carpophores récoltés au pied de ces cèdres de l'Atlas du Parc national de Chréa appartiennent aux genres Cortinarius, Inocybe et Amanita, ce qui laisse présager une certaine spécificité du cèdre de l'Atlas (plante hôte) vis-àvis de son partenaire fongique dont il dépend très fortement pour sa croissance, notamment au stade juvénile.

Niazi (2008) a montré que le champignon Amanita vaginata forme avec Pinus wallichiana des ectomycorhizes dichotomiques à corraloïdes tandis qu'avec Cortinarius hinnuleus il forme des monopodiales pennées à monopodiales pyramidales. Par contre, Inocybe nitidiuscula forme des ectomycorhizes dichotomiques (Ilyas et al., 2012). En tenant compte uniquement des genres des champignons ectomycorhiziens
(Cortinarius hinnuleus, Inocybe nitidiuscula) qui ont des liens phylétiques avec les espèces Cortinarius cedretorum et Inocybe geophylla, une certaine similitude des formes apparaît entre les ectomycorhizes de Pinus wallichiana, conifère des montagnes du Pakistan, et celles du cèdre de l'Atlas, conifère des montagnes d'Afrique du Nord.

À l'échelle cellulaire, l'inoculation ectomycorhizienne a généré la formation du manteau fongique entourant les racines et le réseau de Hartig. Les résultats des coupes anatomiques ont révélé la présence de ces deux traits de la symbiose ectomycorhizienne, ainsi que la réussite de la mycorhization avec les inocula sporaux en conditions expérimentales de la serre.

Le réseau de Hartig résulte de la pénétration plus ou moins profonde d'hyphes mycéliens issus du manteau entre les cellules de la première assise épidermique des racines courtes de la plante hôte. Ces hyphes constituent un vaste réseau mycélien, qui se nourrit exclusivement de matières organiques des horizons supérieurs du sol. Ils fructifient à ce niveau pour former des carpophores (Dommergues et al., 1999).

Les inocula sporaux utilisés ont affecté positivement la nutrition phosphatée et azotée : les aiguilles des plantules du cèdre inoculées ont accumulé des concentrations nettement plus élevées en phosphore et en azote comparativement à celles des plantules témoins. En effet, l'augmentation concomitante des teneurs en $\mathrm{P}$ et $\mathrm{N}$ s'est répercutée sur tous les autres paramètres de croissance mesurés, notamment la masse sèche totale du plant, la longueur de la racine principale et le nombre de racines fines; cette augmentation montre que ceux-ci ont prélevé des quantités plus grandes de ces éléments que les plants de cèdres témoins.

L'augmentation de la croissance du plant de cèdre inoculés laisse donc présager une stimulation de transfert des assimilats vers les racines, ce qui se traduit par une augmentation de la longueur de la racine principale, du nombre de racines fines, ainsi que le développement des mycorhizes et des carpophores des champignons ectomycorhiziens (Lamhamedi et al., 1994 ; Fortin et Lamhamedi, 2009). En effet, les champignons mycorhiziens sont responsables du prélèvement et du transport par la plante des nutriments du sol peu mobiles (magnésium, potassium, phosphore) (Selosse et al., 2006) et mobile comme l'azote (Valtanen et al., 2014).

De nombreux auteurs ont démontré que les mycorhizes améliorent significativement la nutrition en phosphate (Thomson et al., 1994 ; Borges de Souza et al., 2008) et en azote de la plante (Talbot et Treseder, 2010 ; Valtaneen et al., 2014). Par exemple, des augmentations significatives du taux d'azote ont été rapportées chez les plantules inoculées de Pinus halepensis (Rincón et al., 2007) et Picea mariana (Gagnon et al., 1988) comparativement aux plantules témoins non inoculées. Cependant, l'efficacité des mycorhizes dépend particulièrement de l'interaction de la souche fongique et de l'essence forestière (Rincón et al., 2005 ; Kennedy et al., 2007). 


\section{Conclusion}

Nos résultats indiquent clairement que la réussite de la mycorhization des plants du cèdre de l'Atlas en pépinière repose sur l'utilisation d'arbres semenciers, de spores de champignons et du sol organique collectés dans un peuplement représentatif de l'aire naturelle de cette essence. En effet, les spores des champignons ectomycorhiziens se distinguent par leur diversité génétique très élevée et constituent une source d'inoculum moins coûteuse dont les techniques d'application sont à la portée des pépiniéristes. À cet effet, il serait intéressant d'évaluer l'impact des inocula sporaux sur la qualité morpho-physiologique des plants du cèdre de l'Atlas produits dans différentes pépinières forestières, ainsi que sur leur performance dans différents sites de reboisement en relation avec la sécheresse. Il est aussi important d'examiner le devenir des mycobiontes après transplantation car les propriétés physico-chimiques du sol pourraient affecter le degré de mycorhization et leur maintien à long terme (Högberg et Högberg, 2002 ; Garcia et al., 2008).

Parmi les champignons évalués dans la présente étude, à cause de son effet sur l'augmentation de la croissance et de l'amélioration du statut nutritionnel des plants, C. cedretorum pourrait être considéré comme un bon candidat pour une utilisation dans les programmes d'inoculation en pépinière visant à produire des plants mycorhizés de Cedrus atlantica destinés au programme de reboisement en Algérie. Cependant, si l'objectif du programme de reboisement et d'inoculation des plants dans les pépinières forestières consiste à maintenir une diversité d'espèces de champignons ectomycorhiziens dans les sites très dégradés, il serait souhaitable d'utiliser un mélange de spores de ces espèces de champignons ectomycorhiziens dont les carpophores sont dominants sous la cédraie.

\section{Références bibliographiques}

Abourouh M., 1993. Essai de mycorhization en pépinière par les spores de Pisolithus tinctorius. Annales de la Recherche Forestière au Maroc, 26 : 126-137.

Abourouh M., 1995. Les ectomycorhizes du Cèdre de l'Atlas : état des connaissances et perspectives. In : M'Hirit O., Samih A., Malagnoux M. (éds). Le Cèdre de l'Atlas, Cedrus atlantica (Manetti). Actes du Séminaire international sur le Cèdre de l'Atlas, 7-11 juin 1993, Ifrane, Maroc. Annales de la Recherche forestière au Maroc, 27 (1) : 337-348.

Abourouh M., 2000. Ectomycorhizes et mycorhization des principales essences forestières du Maroc. Thèse de doctorat, Faculté des sciences Agdal, Rabat, Maroc, 170 p.

Agerer R., 1995. Colour atlas of ectomycorrhizae. $1^{\text {re }} 9^{\mathrm{e}}$ partie. Schwäbisch-Gmünd, Allemagne, Einhorn-Verlag Eduard Dietenberger $\mathrm{GmbH}$.

Bentouati A., Bariteau M., 2006. Réflexions sur le dépérissement du Cèdre de l'Atlas des Aurès (Algérie). Forêt Méditerranéenne, 4 : 317-322.

Borges de Souza L. A., Bonnassis P. A. P., Filho G. N. S., Lopes de Oliveira V., 2008. New isolates of ectomycorrhizal fungi and the growth of eucalypt. Pesquisa Agropecuária Brasileira, 43 (2): 235-241.

Boukcim H., Conventi S., Mousain D., 2002. Ectomycorhization de Cedrus atlantica en conditions contrôlées : efficacité de deux formes d'inoculum mycélien. Annales des Sciences Forestières, 59 : 839-846.

Chen Y. L., Kang L. H., Malajczuk N., Dell B., 2006. Selecting ectomycorrhizal fungi for inoculating plantations in south China: effect of Scleroderma on colonization and growth of exotic Eucalyptus globulus, E. urophylla, Pinus elliottii, and P. radiata. Mycorrhiza, 16: 251-259.

Conjeaud C., Scehromm P., Mousain D., 1996. Effects of phosphorus and ectomycorrhiza on maritime pine seedlings (Pinus pinaster). New Phytologist, 133: 345-351.

Derridj A., 1990. Étude des populations de Cedrus atlantica M. en Algérie. Thèse de doctorat, Université Paul Sabatier, Toulouse, France, $288 \mathrm{p}$.

Dommergues Y., Duhoux E., Diem H. G., 1999. Les arbres fixateurs de l'azote : Caractéristiques fondamentales et rôle dans l'aménagement des écosystèmes méditerranéens et tropicaux, avec référence particulière aux zones subhumides et arides. Montpellier, France, Espaces 34, $523 \mathrm{p}$.

Duponnois R., Colombet A., Hien V., Thioulouse J., 2005. The mycorrhizal fungus Glomus intraradices and rock phosphate amendment influence plant growth and microbial activity in the rhizosphere of Acacia holosericea. Soil Biology and Biochemistry, 37 (8): 1460-1468.

FAO, Plan Bleu, Silva Mediterranea, FFEM, 2013. État des forêts méditerranéennes 2013. Rome, Italie, FAO, Étude FAO, 207 p.

Fortin J. A., Lamhamedi M. S., 2009. Ecophysiology of sporocarp development of ectomycorrhizal basidiomycetes associated with boreal forest gymnosperms. In: Khasa D., Piché Y., Coughlan A. P. (eds). Advances in mycorrhizal science and technology. Ottawa, Canada, NRC Research Press, 161-173.

Gagnon J., Langlois C. G., Fortin J. A., 1988. Growth and ectomycorrhizal formation of containerized black spruce seedlings as affected by nitrogen fertilization, inoculation type, and symbiont. Revue Canadienne de Recherche Forestière, 18 (7): 922-929. 
Garcia M. O., Ovasapyan T., Greas M., Tresseder K. K., 2008. Mycorrhizal dynamics under elevated $\mathrm{CO}_{2}$ and nitrogen fertilization in a warm temperate forest. Plant and Soil, 303: 301-310.

Guinberteau J., Courtecuisse R., 1997. Diversité des champignons (surtout mycorhiziens) dans les écosystèmes forestiers actuels. Revue Forestière Française, $49:$ 25-39.

Högberg M. N., Högberg P., 2002. Extramatricial ectomycorrhizal mycelium contributes one-third of microbial biomass and produces together with associated roots half the dissolved organic carbon in a forest soil. New Phytologist, 154: 791-795.

Ilyas S., Razaq A., Khalid A. N., 2012. Inocybe nitidiscula and its ectomycorrhizae with Alnus nitida from Galyat, Pakistan. Mycotaxon, 124: 247-54.

Johansen D. A., 1940. Plant microtechnique. New York and London, McGraw-Hill Book Company, 523 p.

John M. K., 1970. Colorimetric determination in soil and plant material with ascorbic acid. Soil Science, 68: 171-177.

Kennedy P. G., Bergemann S. E., Hortal S., Bruns T. D., 2007. Determining the outcome of field-based competition between two Rhizopogon species using real-time PCR. Molecular Ecology, 16: 881-890.

Kope H. H., Fortin J. A., 1990. Germination and comparative morphology of basidiospores of Pisolithus arhizus. Mycologia, 82: 350-357.

Lamhamedi M. S., Fortin J. A., Kope H. H., Kropp B. R., 1990. Genetic variation in ectomycorrhiza formation by Pisolithus arhizus on Pinus pinaster and Pinus banksiana. New Phytologist, 115: 689-697.

Lamhamedi M. S., Fortin J. A., Bernier P. Y., 1991. La génétique de Pisolithus sp. : une approche de biotechnologie forestière pour une meilleure survie des plants en conditions de sécheresse. Sécheresse, $2: 251-258$.

Lamhamedi M. S., Godbout C., Fortin J. A., 1994. Dependence of Laccaria bicolor fruit body development on current photosynthesis of Pinus strobus seedlings. Canadian Journal of Forest Research, 24: 1797-1804.

Lamhamedi M. S., Ammari Y., Fecteau B., Fortin J. A., Margolis H., 2000. Problématique des pépinières forestières en Afrique du Nord et stratégies d'orientation. Cahiers Agricultures, $9: 369-380$.

Lamhamedi M. S., Abourouh M., Fortin J. A., 2009. Technological transfer: The use of ectomycorrhizal fungi in conventional and modern forest tree nursery in northern Africa. In: Khasa D., Piché Y., Coughlan A. P. (eds). Advances in mycorrhizal science and technology. Ottawa, Canada, NRC Research Press, 139-152.

M'Hirit O., 1994. Le Cèdre de l'Atlas (Cedrus atlantica Manetti). Présentation générale et état des connaissances à travers le réseau Silva Mediterranea "Le Cèdre ». Annales de la Recherche Forestière au Maroc, 27 : 5-21.

Marx D. H., 1979. Ectomycorrhizal status of tree seedlings in nurseries and field sites in northern Morocco. Rabat, Maroc, Centre de recherche forestière, Rapport de mission, 8 p.

Mousain D., Plassard C., Argillier C., Sardin T., Leprince F., El Karkouri K. et al., 1994. Stratégie d'amélioration de la qualité des plants forestiers et des reboisements méditerranéens par utilisation de la mycorhization contrôlée en pépinière. Acta Botanica Gallica, $141:$ 571-580.

Mousain D., Falconnet G., Gruez J., Chevalier G., Tillard P., Bousquet N. et al., 1987. Controlled ectomycorrhizal development of mediterranean forest seedlings in the nursery. First results and prospects. In: Sylvia D. M., Hung L. L., Graham J. H. (eds). Mycorrhizae in the next decade. Practical applications and research priorities. Proceedings of the Seventh North American Conference on Mycorrhizae, Gainesville, USA, University of Florida, 129.
Murphy J., Riley J. P., 1962. A modified single solution method for the determination of phosphate in natural waters. Analytica Chimica Acta, 27: 31-35.

Nezzar Hocine H., 1998. Associations mycorhiziennes naturelles de Cedrus atlantica dans le massif du Djurdjura (Algérie) et mycorhization contrôlée. Thèse de doctorat, Université Blaise Pascal, Clermont-Ferrand II, France, $479 \mathrm{p}$.

Niazi A. R., 2008. Biodiversity of ectomycorrhizas in Conifers from Himalaya Moist Temperate Forests of Pakistan. PhD dissertation, Department of Botany, University of the Punja, Lahore, Pakistan.

Olsen S. R., Cole C. V., Watanabe F. S., Dean L. A., 1954. Estimation of available phosphorus in soils by extraction with sodium bicarbonate. USDA, Circular 939, 8 p.

Rincón A., Parladé J., Pera J., 2005. Effects of ectomycorrhizal inoculation and the type of substrate on mycorrhization, growth and nutrition of containerized Pinus pinea L. seedlings produced in commercial nursery. Annals of Forest Science, 62: 1-6.

Rincón A., Parladé J., Pera J., 2007. Influence of the fertilization method in controlled ectomycorrhizal inoculation of two Mediterranean pines. Annals of Forest Science, 64: 577-583.

Rosado S. C. S., Kropp B. R., Piché Y., 1994a. Genetics of ectomycorrhizal symbiosis. I. Host plant variablility and heritability of ectomycorrhizal fungi. New Phytologist, 126: 105-110.

Rosado S. C. S., Kropp B. R., Piché Y., 1994b. Genetics of ectomycorrhizal symbiosis. II. Fungal variablility and heritability of ectomycorrhizal traits. New Phytologist, 126: 111-117.

Sanon K. B., Dianda M., Bazie M., Kone N. G., Bà A., 2011. Effet de l'inoculation avec des spores de champignon ectomycorhizien du genre Scleroderma sur la croissance et la nutrition des plants de Afzelia africana $\mathrm{Sm}$. en pépinière. International Journal of Biological and Chemical Sciences, 5 (5): 1805-1812.

Selosse M. A., Richard F., He X. H., Simard S. W., 2006. Mycorrhizal networks : des liaisons dangereuses. Trends in Ecology and Evolution, 21 (11) : 621-628.

Talbot J. M., Treseder K. K., 2010. Controls over mycorrhizal uptake of organic nitrogen. Pedobiologia, 53 (3): 169-179.

Theodorou C., Bowen G. D., 1987. Germination of basidiospores of mycorrhizal fungi in the rhizosphere of Pinus radiata. New Phytopathology, 106: 217-223.

Thomson B. D., Grove T. S., Malajczuk N., Hardy G. E. St. J., 1994. The effectiveness of ectomycorrhizal fungi in increasing the growth of Eucalyptus globulus Labill. in relation to root colonization and hyphal development in soil. New Phytologist, 126: 517-524.

Torres P., Honrubia M., 1994. Inoculation of containerized Pinus halepensis (Miller) seedlings with basidiospores of Pisolithus arhizus (Pers.) Rauschert, Rhizopogon roseolus (Corda) Th. M. Fr. and Suillus collinitus (Fr.) O. Kuntze. Annals of Forest Science, 51: 521-528.

Valtanen K., Eissfeller V., Beyer F., Hertel D., Scheu S., Polle A., 2014. Carbon and nitrogen fluxes between beech and their ectomycorrhizal assemblage. Mycorrhiza, 24: 645-650.

Wong K. K., Fortin J. A., 1990. Root colonization and intraspecific mycobiont variation in ectomycorrhiza. Symbiosis, 8: 197-231.

Zine El Abidine A., Lamhamedi M. S., Taoufik A., 2013. Relations hydriques des arbres sains et dépérissants de Cedrus atlantica $M$. au Moyen Atlas Tabulaire au Maroc. Géo-Eco-Trop, 37 (2) : 157-176. 


\section{Instructions for authors}

Use these instructions ifyou are preparing a manuscript tosubmit to Bois et Forêts des Tropiques. Potential contributors are advised that careful attention to the details below will greatly assist the Editor and thus speed the processing of their manuscripts. Poorly prepared manuscripts will be returned to authors.

Bois et Forêts des Tropiques publishes papers in the important and wide field of the Tropical and Mediterranean Forests. Papers devoted to the results of original research as well as those which form significant reviews will be considered. Research papers normally should not exceed 6000 words of main text and should have no more than 40 references. Reviews should not exceed 8000 words of main text and should have no more than 80 references A cover letter is needed, showing what was investigated, where and how it was done, why the results are important, what is their innovative nature and transferability, and why the author has chosen Bois et Forêts des Tropiques for his manuscript. Note that it is possible to suggest names for review but also provide names which are not preferred.

Bois et Forêts des Tropiques considers all manuscripts on the strict condition that the manuscript is your own original work, and does not duplicate any other previously published work, in whole or in part, including your own previously published work; the manuscript has been submitted only to Bois et Forêts des Tropiques and is not under consideration or peer review or accepted for publication or in press or published elsewhere; the manuscript contains nothing that is abusive, defamatory, libellous, obscene, fraudulent, or illegal. For all manuscripts non-discriminatory language is mandatory. Sexist or racist terms must not be used.

The Editor will acknowledge receipt of the manuscript, provide it with a manuscript reference number and assign it to reviewers. The reference number of the manuscript should be quoted in all correspondence with Bois et Forêts des Tropiques.

Papers are first inspected for suitability by the Editor or an editorial board member. Those suitable papers are then critically reviewed by usually two or three expert persons. On their advice the Editor provisionally accepts, or rejects, the paper. If acceptance is indicated the manuscript is usually returned to the author for revision. In some cases a resubmission is invited and on receipt of the new version, the paper may be sent to a third referee. If the author does not return the revised or resubmitted version within 60 days the paper will be classified as rejected. Final acceptance is made when the manuscript has been satisfactorily revised.

\section{General guidelines}

Manuscripts are accepted in French or English. For English manuscripts, British English spelling and punctuation are preferred. Manuscripts should be prepared according to the following structure:

Page 1. Title page. This should contain (a) the full title, preferably of less than 15 words and usually containing the geographical location of the study; (b) a running title of not more than 48 letters and spaces; (c) a list of up to 8 key words, separated by commas, in alphabetical order suitable for international retrieval systems;

Page 2. Authors page. The page should contain full names, affiliations (department, university), postal addresses, telephone numbers and email addresses of each author. Please provide an institutional email address for each author. One author should be identified as the corresponding author.

Page 3. Abstract. This should be a single paragraph, in passive mode, no more than 275 words long, concise summary of the paper intelligible on its own in conjunction with the title, without abbreviations or references. The abstract is written in the same language used in the text.

Page 4. et sea. Text. The main body of the text may contain the following sections in the sequence indicated: (a) Introduction, (b) Methods, (c) Results, (d) Discussion, (e) Conclusion, (f) Acknowledgements, (g) Literature Cited, (h) Appendices (as appropriate), (i) Tables with caption(s), (j) figure caption(s) as a list, (k) photograph captions as a list.

Tables (preferably in MS Word, they must not be submitted as images) should be provided either at the end of the manuscript or as separate files. Tables should be numbered consecutively with Latin numerals (for example: table I) and every table should be cited at least once in the text, in consecutive order.

Figures should be submitted as separate files in TIF or EPS format but captions to figures should be supplied on a separate sheet at the end of the main manuscript. All figures must be cited in consecutive order and are numbered consecutively with Arabic numerals (for example: figure 1). The actual figures should be supplied in a separate file. Ensure you have permission to use any figures or photos you are reproducing from another source. In addition, provide in separate Excel files the source of the graphics, to enhance figures during the publication process.

Photos are strongly recommended. Their number should range from 3 to 6 . They must be numbered, with Arabic numerals (for example: photo 1), in the order in which they appear in the text. Photograph files are in $10 \mathrm{~cm} \times 15 \mathrm{~cm}$ format, with a minimal resolution of $300 \mathrm{dpi}$, and must be compressed into JPEG at maximum quality.

Tables, figures and photos: Indicate in the text where the tables and figures should appear, for example by inserting [Table I near here]. All the captions have to be informative and explicit. Acknowledgements should be brief. Please supply all details required by any funding and grant-awarding bodies.

Notes should be avoided if at all possible; any notes will be printed as footnotes and not at the end of the paper.

Scientific names - The complete Latin name (genus, species and authority) must be given in full for every organism when first mentioned in the text unless a standard nomenclatural reference is available which can be cited. Authorities might alternatively appear in Tables where they are first used. Names of taxa at generic rank and below should be in italics.

\section{Advice to authors on preparing a manuscript}

Font: Times New Roman, 12 point. Double spacing must also be used throughout, allowing wide margins (about $3 \mathrm{~cm}$ ) on all sides. Main text pages are numbered. All pages of text have line numbers.

Section headings should be concise.

The corresponding author must be authorized by all co-authors to act as an agent on their behalf in all matters pertaining to publication of the manuscript, and the order of names should be agreed by all authors.

Anonymity for peer review: Ensure your identity and that ofyour co-authors is not revealed in the text of your article or in your manuscript files when submitting the manuscript for review. Headings: Please indicate the level of the section headings in your article.

If your article is accepted for publication, it will be copy-edited and typeset in the correct style for the journal.

\section{References style}

In the text

One author: Smith (2014) or (Smith, 2014)

Two authors: Smith and Jones (2014) or (Smith and Jones, 2014)

Three or more authors: Dupondt et al. (2014) or (Dupondt et al., 2015)

When several in-text references occur at the same point, give them in chronological order separated by semicolons.

Reference list

At the end of a document, list the references to sources that have been cited within the text, including those found in tables and figures, under the heading References.

To speed the processing of the manuscript, we strongly recommend the authors to check very carefully reference list $v s$ references in the text and tables.

References with two or more authors should be arranged first alphabetically then chronologically. The names of cited journals should be given in full.

Book: Smith A. J., 2012. Forest ecosystems. Abingdon, Routledge, 498 p.

Chapter: Smith A. J, Jones B. D., 2014. Hawaii new forest ecosystems. In: Tropical new forests, Eds McKey D. M. and Willey E. M. Abingdon, Routledge, p. 229-244.

Journal: Smith A. J, Jones B. D., 2014. Reconsidering tropical forestry. Forestry Journal, 62, 4:112-117.

If six or more authors, list the first six followed by a comma and et al.

\section{Figures}

Please provide the highest quality figure format possible. Please be sure that all imported scanned material is scanned at the appropriate resolution: $1200 \mathrm{dpi}$ for line art, $600 \mathrm{dpi}$ for grayscale and $300 \mathrm{dpi}$ for color.

Please do not embed figures in the manuscript file.

Files should be saved as one of the following formats: XLS (MS Excel file), TIFF (tagged image file format), PostScript or EPS (encapsulated PostScript).

All figures must be numbered in the order in which they appear in the manuscript (e. g. figure 1, figure 2). In multi-part figures, each part should be labelled (e. g. figure 1(a), figure 1(b)). Figure captions must be saved separately, as part of the file containing the complete text of the manuscript, and numbered correspondingly.

\section{Reproduction of copyright material}

If you wish to include any material in your manuscript in which you do not hold copyright, you must obtain written permission from the copyright owner, prior to submission. This applies to direct (verbatim or facsimile) reproduction as well as "derivative reproduction" (where you have created a new figure or table which derives substantially from a copyrighted source).

\section{Manuscript submission}

All submissions should be made using the e-mailing address of Bois et Forêts des Tropiques 0. Please note that the total size of the attached files should not exceed 7 Mo. If the whole size is higher, please use separate mailing.

Manuscripts may be submitted in any standard editable format, including Word and EndNote.

\section{Copyright and authors' rights}

To assure the integrity, dissemination, and protection against copyright infringement of published articles, you will be asked to assign us, via a Publishing Agreement, the copyright in your article. Your Article is defined as the final, definitive, and citable Version of Record, and includes the accepted manuscript in its final form, including the abstract, text, bibliography, and all accompanying tables, illustrations, data. Our Publishing Agreement with you will constitute the entire agreement and the sole understanding between you and us; no amendment, addendum, or other communication will be taken into account when interpreting your and our rights and obligations under this Agreement.

For further information, or to submit an article, please contact: E-mail: bft@cirad.fr

bft.cirad.fr 